\title{
Generalized quantum tomographic maps
}

\author{
M. Asorey, ${ }^{1}$ P. Facchi, ${ }^{2,3}$ V.I. Man'ko, ${ }^{4}$ G. Marmo,${ }^{5,6}$ S. Pascazio, ${ }^{7,3}$ and E. C. G. Sudarshan ${ }^{8}$ \\ ${ }^{1}$ Departamento de Física Teórica, Facultad de Ciencias, \\ Universidad de Zaragoza, 50009 Zaragoza, Spain \\ ${ }^{2}$ Dipartimento di Matematica and MECENAS, Università di Bari, I-70125 Bari, Italy \\ ${ }^{3}$ INFN, Sezione di Bari, I-70126 Bari, Italy \\ ${ }^{4}$ P.N. Lebedev Physical Institute, Leninskii Prospect 53, Moscow 119991, Russia \\ ${ }^{5}$ Dipartimento di Scienze Fisiche and MECENAS, \\ Università di Napoli "Federico II", I-80126 Napoli, Italy \\ ${ }^{6}$ INFN, Sezione di Napoli, I-80126 Napoli, Italy \\ ${ }^{7}$ Dipartimento di Fisica and MECENAS, Università di Bari, I-70126 Bari, Italy \\ ${ }^{8}$ Department of Physics, University of Texas, Austin, Texas 78712, USA
}

(Dated: July 4, 2018)

\begin{abstract}
Some non-linear generalizations of classical Radon tomography were recently introduced by $\mathrm{M}$. Asorey et al [Phys. Rev. A 77, 042115 (2008)], where the straight lines of the standard Radon map are replaced by quadratic curves (ellipses, hyperbolas, circles) or quadratic surfaces (ellipsoids, hyperboloids, spheres). We consider here the quantum version of this novel non-linear approach and obtain, by systematic use of the Weyl map, a tomographic encoding approach to quantum states. Non-linear quantum tomograms admit a simple formulation within the framework of the starproduct quantization scheme and the reconstruction formulae of the density operators are explicitly given in a closed form, with an explicit construction of quantizers and dequantizers. The role of symmetry groups behind the generalized tomographic maps is analyzed in some detail. We also introduce new generalizations of the standard singular dequantizers of the symplectic tomographic schemes, where the Dirac delta-distributions of operator-valued arguments are replaced by smooth window functions, giving rise to the new concept of thick quantum tomography. Applications for quantum state measurements of photons and matter waves are discussed.
\end{abstract}

PACS numbers: 03.65.Wj; 42.30.Wb; 02.30.Uu

\section{INTRODUCTION}

Quantum tomography has a long and interesting history. The first intuition, hinting at a tomographic reconstruction of quantum states, dates back to 1933, when Pauli asked whether it is possible to uniquely associate quantum states with probability distributions, as in classical statistical mechanics [1, 2]. Pauli's observation was, in fact, more subtle and articulated, as he wondered whether two given position and momentum probability distribution functions are mathematically and physically compatible, and whether it is possible to unambiguously reconstruct the quantum state (the wave function) from their knowledge. The answer to Pauli's original question is negative [3, 4], but his general idea to associate quantum states with a set of probability distribution functions eventually led to the basic formulation of quantum tomography.

The tomographic representation of quantum states is based on the Radon transform [5] of the Wigner quasiprobability distribution function [6]. The application to the reconstruction of quantum states was pioneered in the 80's [7, 8] and led to the first experimental verifications in the 90's [9 11]. Since then, the tomographic approach to the analysis of quantum states has become a booming and consolidated field of investigation [12, 13], leading to theoretical ideas and experimental proposals [14 22], thanks also to its important applications in quantum information [23, 24].
The original Radon transform [5] maps functions defined on a two-dimensional plane onto functions defined on a two-dimensional cylinder. The key feature is that the transform is invertible. There exist several important generalizations of the Radon transform (see, e.g., 25 27]). More recent analysis have focused on symplectic transforms [28], on the deep relationship with classical systems and classical dynamics 29, [30], on the formalism of star product quantization 31] and on the study of marginals along curves that are not straight lines [32].

The aim of this work is to study generalizations of the Radon transform to multidimensional phase spaces and to frameworks based on marginals along curves or surfaces described by quadratic equations. We shall study applications to both classical and quantum systems. For classical systems we use the Radon transform of the probability densities in phase space of a classical particle and build the corresponding tomographic map into Radon components of the initial probability densities. In quantum systems, we introduce a pair of operators, the density operator and its Radon transform operator, which are related by an invertible map. In the context of Wigner function, these formulas provide an analogy of the Radon transform for classical probability distributions in phase space.

The paper is organized as follows. In Section III we review the symplectic tomographic approach to classical mechanics. In Section III we provide a generalization of symplectic tomography that is based on marginals 
over ellipsoids and hyperboloids and their boundary surfaces, instead of planes and straight lines. The case of marginals based on circles and spheres with shifted centers provides another type of interesting generalization. We also consider, by using a star-product framework, the generalization of Radon transform in operator form for quantum systems in Section IV] Center of mass tomography [33], symplectic tomography and its generalization to several quantum systems is discussed in Section V] using symplectic group Hamiltonians. A generic formulation of tomographic approaches that make use of Hamiltonians which are linear forms in generalized Lie algebras is briefly summarized in this section. In Section VI we consider thick symplectic quantum tomography and compare it with the thick Radon transform in Section VII] Finally, some conclusions and perspectives are conveyed in Section VIII.

\section{SYMPLECTIC QUANTUM TOMOGRAPHY}

Let $\hat{\rho}$ be a quantum state $\left(\hat{\rho}=\hat{\rho}^{\dagger}, \hat{\rho}>0\right)$. The quantum Radon transform (homodyne tomogram) is given by

$$
\mathcal{R}_{\hat{\rho}}(X, \varphi)=\operatorname{Tr} \hat{\rho} \delta(X \hat{\mathbb{I}}-\hat{q} \cos \varphi-\hat{p} \sin \varphi)
$$

with $\varphi \in[0,2 \pi]$. In terms of the Wigner function

$W(p, q)=\int\left\langle q-\frac{\xi}{2}|\hat{\rho}| q+\frac{\xi}{2}\right\rangle \mathrm{d} \xi, \quad \int W(p, q) \mathrm{d} p \mathrm{~d} q=2 \pi$,

the above expression reads

$$
\begin{aligned}
\mathcal{R}_{\hat{\rho}}(X, \varphi)= & \frac{1}{2 \pi} \int \mathrm{d} p \mathrm{~d} q W(p, q) \\
& \times \delta(X-q \cos \varphi-p \sin \varphi) .
\end{aligned}
$$

The quantum symplectic [34] (or $M^{2}$ [35]) transform is a generalization of the quantum Radon transform (1) and reads

$$
\begin{aligned}
\mathcal{W}_{\hat{\rho}}(X, \mu, \nu) & =\langle\delta(X \hat{\mathbb{I}}-\mu \hat{q}-\nu \hat{p})\rangle_{\rho} \\
& =\operatorname{Tr} \hat{\rho} \delta(X \hat{\mathbb{I}}-\mu \hat{q}-\nu \hat{p}),
\end{aligned}
$$

where $\mu$ and $\nu$ are real parameters. The information content of the two formulations is identical and is expressed by the relation

$$
\mathcal{W}_{\hat{\rho}}(X, r \cos \varphi, r \sin \varphi)=\frac{1}{r} \mathcal{R}_{\hat{\rho}}\left(\frac{X}{r}, \varphi\right),
$$

valid for any $r>0$. Equation (5) is an easy consequence of the fact that the Dirac distribution is positive homogeneous of degree -1 . The two formulations (11) and (4) may differ in practice (the latter being easier to invert [35]) and cease to be equivalent when the Dirac deltafunction is replaced by a finite window function [36]. In the present article, we shall focus on the symplectic version (4).

Equation (4) can be rewritten as the "classical" tomogram of the Wigner function

$$
\mathcal{W}_{\hat{\rho}}(X, \mu, \nu)=\frac{1}{2 \pi} \int W(p, q) \delta(X-\mu q-\nu p) \mathrm{d} p \mathrm{~d} q,
$$

whose inverse transform reads

$$
W(p, q)=\frac{1}{2 \pi} \int \mathcal{W}_{\hat{\rho}}(X, \mu, \nu) \mathrm{e}^{\mathrm{i}(X-\mu q-\nu p)} \mathrm{d} X \mathrm{~d} \mu \mathrm{d} \nu,
$$

or, in terms of the density matrix,

$$
\hat{\rho}=\frac{1}{2 \pi} \int \mathcal{W}_{\hat{\rho}}(X, \mu, \nu) \mathrm{e}^{\mathrm{i}(X \hat{\mathbb{I}}-\mu \hat{q}-\nu \hat{p})} \mathrm{d} X \mathrm{~d} \mu \mathrm{d} \nu .
$$

For completeness and for future convenience, we notice that the inversion formula of the Radon transform (10) is easily obtained from Eqs. (5) and (8) and reads

$$
\begin{aligned}
\hat{\rho}= & \frac{1}{2 \pi} \int \mathrm{d} X \int_{0}^{\infty} \mathrm{d} r \int_{0}^{2 \pi} \mathrm{d} \varphi \mathcal{R}_{\hat{\rho}}(X, \varphi) \\
& \times r \mathrm{e}^{\mathrm{i} r(X \hat{\mathbb{I}}-\hat{q} \cos \varphi-\hat{p} \sin \varphi)} .
\end{aligned}
$$

The generalization of (6) to the multimode case can be achieved in several ways. Let us consider the case of two modes for simplicity. One can either introduce the ordinary tomogram

$$
\begin{aligned}
& \mathcal{W}_{\hat{\rho}}(\vec{X}, \vec{\mu}, \vec{\nu}) \\
& =\left\langle\delta\left(X_{1} \hat{\mathbb{I}}-\mu_{1} \hat{q}_{1}-\nu_{1} \hat{p}_{1}\right) \delta\left(X_{2} \hat{\mathbb{I}}-\mu_{2} \hat{q}_{2}-\nu_{2} \hat{p}_{2}\right)\right\rangle,
\end{aligned}
$$

where $\vec{X}=\left(X_{1}, X_{2}\right), \vec{\mu}=\left(\mu_{1}, \mu_{2}\right)$ and $\vec{\nu}=\left(\nu_{1}, \nu_{2}\right)$, or, alternatively, the "center-of-mass" tomogram

$$
\mathcal{W}_{\mathrm{cm}}(X, \vec{\mu}, \vec{\nu})=\left\langle\delta\left(X \hat{\mathbb{I}}-\mu_{1} \hat{q}_{1}-\nu_{1} \hat{p}_{1}-\mu_{2} \hat{q}_{2}-\nu_{2} \hat{p}_{2}\right)\right\rangle .
$$

The Weyl map (2) provides again the possibility of expressing (10) and (11) as "classical" tomograms

$$
\begin{aligned}
& \mathcal{W}_{\hat{\rho}}(\vec{X}, \vec{\mu}, \vec{\nu})= \frac{1}{(2 \pi)^{2}} \int \mathrm{d} \vec{p} \mathrm{~d} \vec{q} W(\vec{p}, \vec{q}) \\
& \delta\left(X_{1}-\mu_{1} q_{1}-\nu_{1} p_{1}\right) \delta\left(X_{2}-\mu_{2} q_{2}-\nu_{2} p_{2}\right), \\
& \mathcal{W}_{\mathrm{cm}}(X, \vec{\mu}, \vec{\nu})= \frac{1}{(2 \pi)^{2}} \int \mathrm{d} \vec{p} \mathrm{~d} \vec{q} W(\vec{p}, \vec{q}) \\
& \delta\left(X-\mu_{1} q_{1}-\nu_{1} p_{1}-\mu_{2} q_{2}-\nu_{2} p_{2}\right),
\end{aligned}
$$

where $W(\vec{p}, \vec{q})$ is the Wigner function of the two-mode system, normalized to $(2 \pi)^{2}$. 
The inverse transform reads

$$
W(\vec{p}, \vec{q})=\int \mathcal{W}_{\mathrm{cm}}(X, \vec{\mu}, \vec{\nu}) \mathrm{e}^{\mathrm{i}(X-\vec{\mu} \cdot \vec{q}-\vec{\nu} \cdot \vec{p})} \frac{\mathrm{d} X \mathrm{~d} \vec{\mu} \mathrm{d} \vec{\nu}}{(2 \pi)^{2}},
$$

for the center-of-mass tomogram, and

$$
W(\vec{p}, \vec{q})=\int \mathcal{W}_{\hat{\rho}}(\vec{X}, \vec{\mu}, \vec{\nu}) \mathrm{e}^{\mathrm{i}\left(X_{1}+X_{2}-\vec{\mu} \cdot \vec{q}-\vec{\nu} \cdot \vec{p}\right)} \frac{\mathrm{d} \vec{X} \mathrm{~d} \vec{\mu} \mathrm{d} \vec{\nu}}{(2 \pi)^{2}}
$$

for the ordinary symplectic tomogram. The inversion formulae for the density matrix are

$$
\hat{\rho}=\int \mathcal{W}_{\hat{\rho}}(\vec{X}, \vec{\mu}, \vec{\nu}) \mathrm{e}^{\mathrm{i}\left(X_{1} \hat{\mathbb{I}}+X_{2} \hat{\mathbb{I}}-\vec{\mu} \cdot \hat{\vec{q}}-\vec{\nu} \cdot \hat{\vec{p}}\right)} \frac{\mathrm{d} \vec{X} \mathrm{~d} \vec{\mu} \mathrm{d} \vec{\nu}}{(2 \pi)^{2}},
$$

for the symplectic case, and

$$
\hat{\rho}=\int \mathcal{W}_{\mathrm{cm}}(X, \vec{\mu}, \vec{\nu}) \mathrm{e}^{\mathrm{i}(X \hat{\mathbb{I}}-\vec{\mu} \cdot \hat{\vec{q}}-\vec{\nu} \cdot \hat{\vec{p}})} \frac{\mathrm{d} X \mathrm{~d} \vec{\mu} \mathrm{d} \vec{\nu}}{(2 \pi)^{2}}
$$

for the center-of-mass tomography. The advantage of the symplectic tomogram is that it permits the splitting into subsystems for separable states, which is not possible in the center-of-mass tomogram. This property might be very relevant for the fast reconstruction of non-entangled states.

In the case of $M$ subsystems with their own centers of mass, tomography is defined by

$$
\begin{array}{r}
\mathcal{W}_{\mathrm{cm}}\left(X_{1}, \vec{\mu}_{1}, \vec{\nu}_{1}, X_{2}, \vec{\mu}_{2}, \vec{\nu}_{2}, \ldots, X_{M}, \vec{\mu}_{M}, \vec{\nu}_{M}\right) \\
=\left\langle\prod_{i=1}^{M} \delta\left(X_{i} \hat{\mathbb{I}}-\vec{\mu}_{i} \cdot \hat{\vec{q}}_{i}-\vec{\nu}_{i} \cdot \hat{\vec{p}}_{i}\right)\right\rangle,
\end{array}
$$

where $\vec{\mu}_{i}=\left(\mu_{i 1}, \mu_{i 2}, \ldots, \mu_{i N_{i}}\right), \vec{\nu}_{i}=\left(\nu_{i 1}, \nu_{i 2}, \ldots, \nu_{i N_{i}}\right)$, with $i=1,2, \ldots, M$ and

$$
\sum_{i=1}^{M} N_{i}=N .
$$

The inversion formula is given by

$$
\begin{aligned}
\hat{\rho}= & \int \mathcal{W}_{\mathrm{cm}}\left(X_{1}, \overrightarrow{\mu_{1}}, \overrightarrow{\nu_{1}}, X_{2}, \vec{\mu}_{2}, \vec{\nu}_{2}, \ldots, X_{M}, \vec{\mu}_{M}, \vec{\nu}_{M}\right) \\
& \mathrm{e}^{\mathrm{i} \sum_{j}\left(X_{j} \hat{\mathbb{1}}-\vec{\mu}_{j} \cdot \hat{\vec{\mu}}_{j}-\vec{\nu}_{j} \cdot \hat{\vec{p}}_{j}\right)} \frac{\mathrm{d} \vec{X} \mathrm{~d} \vec{\mu} \mathrm{d} \vec{\nu}}{(2 \pi)^{M}} .
\end{aligned}
$$

The symplectic tomogram can also be constructed in the case of multimodal systems.

\section{QUADRATIC TOMOGRAMS}

One of the main objectives of this article is to study generalizations of the quantum Radon transform to marginals along curves or surfaces described by quadratic, rather than linear equations. The solution of the classical problem was obtained in Ref. [32]. Given an
$M \times M$ symmetric operator $B$ and two $M$-dimensional vectors $\vec{x}$ and $\vec{\alpha}$, let

$$
X=(\vec{x}-\vec{\alpha}) \cdot B(\vec{x}-\vec{\alpha}),
$$

where $\vec{x} \cdot \vec{y}$ denotes the scalar product of vectors $\vec{x}$ and $\vec{y}$. The classical tomogram of a function $f(\vec{x})$ reads

$$
\omega_{f}(X, \vec{\alpha})=\int_{\mathbb{R}^{m}} f(\vec{x}) \delta(X-(\vec{x}-\vec{\alpha}) \cdot B(\vec{x}-\vec{\alpha})) \mathrm{d} \vec{x} .
$$

Observe that the $\delta$-function is supported on the quadrics (ellipsoids, hyperboloids, etc., that can also have degeneracies) defined by Eq. (20). The inverse map is

$$
\begin{gathered}
f(\vec{x})=\frac{|\operatorname{det} B|}{\pi^{m}} \int_{\mathbb{R}^{m+1}} \mathrm{~d} X \mathrm{~d} \vec{\alpha} \omega_{f}(X, \vec{\alpha}) \\
\times \mathrm{e}^{\mathrm{i}(X-(\vec{x}-\vec{\alpha}) \cdot B(\vec{x}-\vec{\alpha}))} .
\end{gathered}
$$

The solution of the quantum problem is similar. With a slight abuse of terminology, we shall speak of multidimensional phase spaces and quadratic "Hamiltonians" of the type

$$
\hat{H}=\frac{1}{2} \hat{\vec{Q}} \cdot B \hat{\vec{Q}}+\vec{C} \cdot \hat{\vec{Q}}
$$

where

$$
\hat{Q}_{j}=\hat{p}_{j}, \quad \hat{Q}_{N+j}=\hat{q}_{j}, \quad(j=1, \ldots, N),
$$

are the momentum and position operators, and $\vec{C}$ and $B$ denote an $2 N$-dimensional vector and a $2 N \times 2 N$ symmetric matrix, which parameterize the different types of the quadratic Hamiltonian. Observe that since we are working in a symplectic framework, $M \rightarrow 2 N$.

The quantum counterpart of the classical tomogram (21) reads

$$
\mathcal{W}(X, \vec{\mu}, \vec{\nu})=\left\langle\delta\left(X \hat{\mathbb{I}}-\hat{\mathbb{H}}_{\vec{\mu} \vec{\nu}}\right)\right\rangle
$$

where

$$
\hat{\mathbb{H}}_{\vec{\mu} \vec{\nu}}=\frac{1}{2}(\hat{\vec{Q}}-\vec{r}) \cdot B(\hat{\vec{Q}}-\vec{r})+\vec{C} \cdot(\hat{\vec{Q}}-\vec{r})
$$

and $\vec{r}=(\vec{\mu}, \vec{\nu})$ is a $2 N$-dimensional vector. The coordinate $X$ plays the role of "energy". The Hamiltonian can be degenerate, depending on the type of eigenvalues of the $2 N \times 2 N$ symmetric matrix $B[36]$. The parameters $\vec{\mu}$ and $\vec{\nu}$ have the meaning of shift parameters of the centers of the operatorial quadratic curves (surfaces).

From the Hamiltonian (26) one can derive a quantum tomographic map similar to that obtained in the classical case, by using the Wigner function:

$$
\mathcal{W}(X, \vec{\mu}, \vec{\nu})=\int \frac{\mathrm{d} \vec{p} \mathrm{~d} \vec{q}}{(2 \pi)^{N}} \delta\left(X-\mathbb{H}_{\vec{\mu} \vec{\nu}}\right) W(\vec{q}, \vec{p}),
$$


where the Hamiltonian $\mathbb{H}_{\vec{\mu} \vec{\nu}}$ is given by (26) with the operators $\hat{\vec{q}}$ and $\hat{\vec{p}}$ replaced by c-numbers $\vec{q}$ and $\vec{p}$. Since Eq. (27) is equivalent to Eq. (21), the reconstruction function follows directly from Eq. (22) with the replacement $B \rightarrow \frac{1}{2} B$, namely,

$$
\hat{\rho}=\int \mathcal{W}(X, \vec{\mu}, \vec{\nu}) \mathrm{e}^{\mathrm{i}\left(X \hat{\mathbb{I}}-\hat{\mathbb{H}}_{\vec{\mu} \vec{\nu}}\right)}|\operatorname{det} B| \frac{\mathrm{d} X \mathrm{~d} \vec{\mu} \mathrm{d} \vec{\nu}}{(2 \pi)^{N}} .
$$

The generalization to multipartite systems is straightforward. If we have $M$ subsystems we can define the multipartite tomogram

$$
\begin{aligned}
& \mathcal{W}_{\mathrm{cm}}(\vec{X}, \vec{\mu}, \vec{\nu})=\int W(\vec{q}, \vec{p}) \prod_{j=1}^{M} \delta\left(X_{j} \hat{\mathbb{I}}-\hat{\mathbb{H}}_{\vec{\mu}_{j} \vec{\nu}_{j}}\right) \frac{\mathrm{d} \vec{p}_{j} \mathrm{~d} \vec{q}_{j}}{(2 \pi)^{N_{j}}} \\
& \vec{X}=\left(X_{1}, X_{2}, \ldots, X_{M}\right), \quad \vec{\mu}=\left(\vec{\mu}_{j}\right), \quad \vec{\nu}=\left(\vec{\nu}_{j}\right),
\end{aligned}
$$

whose inverse transform is

$$
\begin{aligned}
\hat{\rho}= & \int \mathcal{W}_{\mathrm{cm}}(\vec{X}, \vec{\mu}, \vec{\nu}) \prod_{j=1}^{M} \mathrm{e}^{\mathrm{i}\left(X_{j} \hat{\mathbb{I}}-\hat{\mathbb{H}} \vec{\mu}_{j} \vec{\nu}_{j}\right)} \\
& \times\left|\operatorname{det} B_{j}\right| \frac{\mathrm{d} X_{j} \mathrm{~d} \vec{\mu}_{j} \mathrm{~d} \vec{\nu}_{j}}{(2 \pi)^{N_{j}}} .
\end{aligned}
$$

\section{STAR-PRODUCT FRAMEWORK}

It is interesting to see how the transform with quadratic curves in phase space can be written in the form of a star-product quantization 31. Any starproduct scheme is aimed at constructing a bijective map of operators $\hat{A}$ acting on a Hilbert space $\mathcal{H}$ and the space of functions $f_{A}$ defined on a manifold $\mathcal{M}$. The map can be constructed in terms of two operator-valued functions $\hat{D}$ and $\hat{U}$ defined on $\mathcal{M}$ and called "quantizers" and "dequantizers", respectively. These two functions satisfy the identity

$$
\operatorname{Tr}(\hat{D}(x) \hat{U}(y))=\delta(x-y)
$$

for every $x, y \in \mathcal{M}$. The map is then defined by the formula

$$
f_{A}(x)=\operatorname{Tr}(\hat{A} \hat{U}(x))
$$

and the inverse map is defined by

$$
\hat{A}=\int f_{A}(x) \hat{D}(x) \mathrm{d} x .
$$

The star product is defined on the space of functions $f_{A}$ by

$$
f_{A} \star f_{B}=f_{A B}
$$

The kernel of this associative star product is then given by

$$
K\left(x_{1}, x_{2}, x_{3}\right)=\operatorname{Tr}\left(\hat{D}\left(x_{1}\right) \hat{D}\left(x_{2}\right) \hat{U}\left(x_{3}\right)\right)
$$

and satisfies

$$
f_{A} \star f_{B}(x)=\int f_{A}\left(x_{1}\right) f_{A}\left(x_{2}\right) K\left(x_{1}, x_{2}, x\right) \mathrm{d} x_{1} \mathrm{~d} x_{2} .
$$

The key observation is that the tomogram can be interpreted in terms of a dequantizer

$$
\hat{U}(X, \vec{\mu}, \vec{\nu})=\delta\left(X \hat{\mathbb{I}}-\hat{\mathbb{H}}_{\vec{\mu} \vec{\nu}}\right),
$$

and a quantizer

$$
\hat{D}(X, \vec{\mu}, \vec{\nu})=\frac{|\operatorname{det} B|}{(2 \pi)^{N}} \mathrm{e}^{\mathrm{i}\left(X \hat{\mathbb{I}}-\hat{\mathbb{H}}_{\vec{\mu} \vec{\nu}}\right)}
$$

Thus, for an arbitrary observable $\hat{A}$, one can introduce the quadratic tomogram

$$
\mathcal{W}_{A}(X, \vec{\mu}, \vec{\nu})=\operatorname{Tr} \hat{A} \delta\left(X \hat{\mathbb{I}}-\hat{\mathbb{H}}_{\vec{\mu} \vec{\nu}}\right)
$$

Also one can reconstruct the observable operator from its quadratic tomogram

$$
\hat{A}=\int \mathcal{W}_{A}(X, \vec{\mu}, \vec{\nu}) \hat{D}(X, \vec{\mu}, \vec{\nu}) \mathrm{d} X \mathrm{~d} \vec{\mu} \mathrm{d} \vec{\nu} .
$$

There exist also tomograms based on shifts of quadratic curves in phase space, but, as in Eq. (4) for symplectic tomograms, we used instead rescaled position and momentum operators.

One can combine both types of tomograms, derived by shifts and rescaling of variables, obtaining a new class of tomograms, which are the quantum version of the classical tomograms defined by 32 .

$$
\omega_{f}(X, \vec{\mu}, \vec{\nu})=\int_{\mathbb{R}^{2 n}} \delta(X-\vec{\mu} \cdot \vec{q}-\nu(\vec{q}, \vec{p})) f(\vec{q}, \vec{p}) d \vec{q} d \vec{p},
$$

where $\vec{p}$ and $\vec{q}$ are vectors in $\mathbb{R}^{n}$ and

$$
\nu(\vec{q}, \vec{p})=\sum_{j=1}^{n} \nu_{j} q_{j} p_{j}
$$

This map corresponds to a deformation of the standard multidimensional Radon transform by means of the following diffeomorphism of $\mathbb{R}^{2 n} \backslash \bigcup_{j}\left\{(\vec{q}, \vec{p}): q_{j}=0\right\}$

$$
\left(q_{i}, p_{j}\right) \mapsto\left(x_{i}, y_{j}\right)=\left(q_{i}, q_{j} p_{j}\right),
$$

whose Jacobian is

$$
J(\vec{q}, \vec{p})=\left|\frac{\partial(\vec{x}, \vec{y})}{\partial(\vec{q}, \vec{p})}\right|=\prod_{j=1}^{n}\left|q_{j}\right| .
$$

The inverse map is given by

$$
\begin{array}{r}
f(\vec{q}, \vec{p})=\int_{\mathbb{R}^{2 n+1}} \frac{d X d \vec{\mu} d \vec{\nu}}{(2 \pi)^{2 n}} \omega_{f}(X, \vec{\mu}, \vec{\nu}) \\
\times \prod_{j=1}^{n}\left|q_{j}\right| \mathrm{e}^{i(X-\vec{\mu} \cdot \vec{q}-\nu(\vec{q}, \vec{p}))} .
\end{array}
$$


This corresponds to the higher-dimensional generalization of the Bertrand-Bertrand tomography [7].

The quantum extension is straightforward. One introduces the Hamiltonian

$$
\hat{\mathbb{H}}_{\xi \nu}(\hat{\vec{q}}, \hat{\vec{p}})=\sum_{i=1}^{N} \xi_{i} \hat{q}_{i}+\frac{1}{2} \sum_{j=1}^{N} \nu_{j}\left(\hat{q}_{j} \hat{p}_{j}+\hat{p}_{j} \hat{q}_{j}\right) .
$$

The dequantizer operator

$$
\hat{U}(X, \vec{\xi}, \vec{\nu})=\delta\left(X \hat{\mathbb{I}}-\hat{\mathbb{H}}_{\vec{\xi} \vec{\nu}}(\hat{\vec{q}}, \hat{\vec{p}})\right)
$$

yields the quantum tomogram

$$
\mathcal{W}(X, \vec{\xi}, \vec{\nu})=\operatorname{Tr} \hat{\rho} \delta\left(X \hat{\mathbb{I}}-\hat{\mathbb{H}}_{\vec{\xi} \vec{\nu}}(\hat{\vec{q}}, \hat{\vec{p}})\right)
$$

and corresponds to the quantizer

$$
\hat{D}(X, \vec{\xi}, \vec{\nu})=\prod_{j=1}^{N}\left|\hat{q}_{j}\right| \mathrm{e}^{\mathrm{i}\left(X \hat{\mathbb{I}}-\hat{\mathbb{H}}_{\vec{\xi} \vec{\nu}}(\hat{\vec{q}}, \hat{\vec{p}})\right)}
$$

This permits to recover the quantum state from its tomogram. The modulus of the operator is defined by the Weyl quantization of the symbol $\left|q_{j}\right|$.

\section{GROUP HAMILTONIANS}

The applications considered so far involve position and momentum coordinates. In the linear and quadratic case, the Hamiltonians from which we constructed the quantizers and dequantizers can be written in the form

$$
\hat{H}_{g_{\alpha}}=\sum_{\alpha}\left(g_{o}\right)_{\alpha} \hat{L}_{\alpha}
$$

where $\hat{L}_{\alpha}$ are generators of Lie groups. In the examples considered in this article, they belong to representations of the inhomogeneous Lie group $\operatorname{ISp}(2 N, \mathbb{R})$ and its subgroups. The group $\operatorname{ISp}(2 N, \mathbb{R})$ is defined as the group of affine transformations of $\mathbb{R}^{2 N} \equiv T^{*} \mathbb{R}^{N}$ which preserve its natural symplectic form. It the semi-direct product of the translation group and the linear symplectic group. The reason for this choice is because this group acts on the Heisenberg-Weyl group as a group of automorphisms. Within this framework, the delta function is expressed as

$$
\delta\left(X \hat{\mathbb{I}}-\sum_{\alpha}\left(g_{o}\right)_{\alpha} \hat{L}_{\alpha}\right)=\frac{1}{2 \pi} \int \mathrm{d} t \mathrm{e}^{\mathrm{i} t\left(X \hat{\mathbb{I}}-\sum_{\alpha}\left(g_{o}\right)_{\alpha} \hat{L}_{\alpha}\right)}
$$

and

$$
\hat{U}(g(t))=\mathrm{e}^{-\mathrm{i} t\left(\sum_{\alpha}\left(g_{o}\right)_{\alpha} \hat{L}_{\alpha}\right)}
$$

is the operator representation of the Lie group.
Thus, the tomogram can be rewritten in a form that only depends on group element parameters $\left(g_{0}\right)_{\alpha}$ and the Radon variable $X$ :

$$
\begin{aligned}
\mathcal{W}\left(X,\left(g_{0}\right)_{\alpha}\right) & =\left\langle\delta\left(X \hat{\mathbb{I}}-\sum_{\alpha}\left(g_{o}\right)_{\alpha} \hat{L}_{\alpha}\right)\right\rangle \\
& =\frac{1}{2 \pi} \operatorname{Tr} \int \mathrm{d} t \hat{\rho} \mathrm{e}^{\mathrm{i} t X} \hat{U}(g(t)) \\
& =\frac{1}{2 \pi} \int \mathrm{d} t \mathrm{e}^{\mathrm{i} t X} \operatorname{Tr}(\hat{\rho} \hat{U}(g(t))) .
\end{aligned}
$$

Therefore, the tomogram is related to the orbit of the group and the Fourier integral of the trace of the orbit in the group representation. This group-theoretical representation permits the extension to more general cases [37.

\section{THICK QUANTUM TOMOGRAPHY}

We now turn our attention to thick tomographic maps [36], which is a more realistic approach for practical applications, because instead of marginals defined over lines, as in the classical Radon transform [5] or quadrics as in the quadratic generalized Radon transform [30, 32], it involves a thick window function $\Xi$. This is convoluted with the tomographic map and concentrates the marginals around some given background curves (that can be lines or quadrics), without resorting to a singular delta function. For example, if the weight function $\Xi$ is a step function, it defines marginals along thick lines or thick quadratic curves. In the quantum case, this amounts to replacing in the definition of the dequantizer $\hat{U}(x)$ the Dirac delta-function by the weight function $\Xi$,

$$
\hat{U}\left(X,\left(g^{0}\right)_{\alpha}\right)=\Xi\left(X \hat{\mathbb{I}}-\sum_{\alpha}\left(g_{o}\right)_{\alpha} \hat{L}_{\alpha}\right) .
$$

For the symplectic quantum tomography, one has the dequantizer

$$
\hat{U}(X, \mu, \nu)=\Xi(X \hat{\mathbb{I}}-\mu \hat{q}-\nu \hat{p}) .
$$

The new tomogram reads

$$
\mathcal{W}_{\Xi}(X, \mu, \nu)=\operatorname{Tr} \hat{\rho} \Xi(X \hat{\mathbb{I}}-\mu \hat{q}-\nu \hat{p}),
$$

Using the Weyl map one obtains a tomogram for the Wigner function

$$
\mathcal{W}_{\Xi}(X, \mu, \nu)=\frac{1}{2 \pi} \int W(p, q) \Xi(X-\mu q-\nu p) \mathrm{d} p \mathrm{~d} q .
$$

The interesting property of the above formula (57) is that it can be inverted in complete analogy with the classical thick tomography introduced in [36]. The thick tomogram can be expressed in terms of standard symplectic tomograms via a convolution formula

$$
\mathcal{W}_{\Xi}(X, \mu, \nu)=\int \mathcal{W}(Y, \mu, \nu) \Xi(X-Y) \mathrm{d} Y,
$$


which leads to the explicit construction of the inverse transform. Indeed, the inverse transform is obtained by means of a Fourier transform of the convolution integral

$$
W(p, q)=\frac{\mathcal{N}_{\Xi}}{2 \pi} \int \mathcal{W}_{\Xi}(X, \mu, \nu) \mathrm{e}^{\mathrm{i}(X-\mu q-\nu p)} \mathrm{d} X \mathrm{~d} \mu \mathrm{d} \nu,
$$

where

$$
\mathcal{N}_{\Xi}=\frac{1}{\widetilde{\Xi}(-1)}, \quad \widetilde{\Xi}(-1)=\int \Xi(z) \mathrm{e}^{\mathrm{i} z} \mathrm{~d} z
$$

In invariant form, the state reconstruction is achieved by

$$
\hat{\rho}=\frac{\mathcal{N}_{\Xi}}{2 \pi} \int \mathcal{W}_{\Xi}(X, \mu, \nu) \mathrm{e}^{\mathrm{i}(X \mathbb{I}-\mu \hat{q}-\nu \hat{p})} \mathrm{d} X \mathrm{~d} \mu \mathrm{d} \nu .
$$

The quantizer operator in thick symplectic tomography is

$$
\hat{D}(X, \mu, \nu)=\frac{\mathcal{N}_{\Xi}}{2 \pi} \mathrm{e}^{\mathrm{i}(X \mathbb{I}-\mu \hat{q}-\nu \hat{p})} .
$$

Let us now consider a particular example of thick tomogram to illustrate the potentialities of the new method. If the weight function is a gaussian function

$$
\Xi(z)=\frac{1}{\sqrt{2 \pi \sigma^{2}}} \mathrm{e}^{-\frac{z^{2}}{2 \sigma^{2}}}
$$

which tends to the delta distribution in the $\sigma \rightarrow 0$ limit,

$$
\lim _{\sigma \rightarrow 0} \Xi(z)=\delta(z),
$$

the thick tomogram of the coherent states $|\alpha\rangle\langle\alpha|$ read

$$
\mathcal{W}_{\sigma}^{\alpha}(X, \mu, \nu)=\frac{1}{\sqrt{\pi\left(\mu^{2}+\nu^{2}+2 \sigma^{2}\right)}} \mathrm{e}^{-\frac{(X-\bar{X})^{2}}{\mu^{2}+\nu^{2}+2 \sigma^{2}}}
$$

where

$$
\bar{X}=\sqrt{2} \mu \operatorname{Re} \alpha+\sqrt{2} \nu \operatorname{Im} \alpha .
$$

For the vacuum state $|0\rangle\langle 0|$, the tomogram reads

$$
\mathcal{W}_{\sigma}^{\mathrm{vac}}(X, \mu, \nu)=\frac{1}{\sqrt{\pi\left(\mu^{2}+\nu^{2}+2 \sigma^{2}\right)}} \mathrm{e}^{-\frac{X^{2}}{\mu^{2}+\nu^{2}+2 \sigma^{2}}} .
$$

The quantizer reads

$$
\hat{D}_{\sigma}(X, \mu, \nu)=\frac{1}{2 \pi} \mathrm{e}^{\frac{\sigma^{2}}{2}+\mathrm{i}(X \mathbb{I}-\mu \hat{q}-\nu \hat{p})}
$$

and the dequantizer is given by

$$
\hat{U}_{\sigma}(X, \mu, \nu)=\frac{1}{\sqrt{2 \pi \sigma^{2}}} \mathrm{e}^{-\frac{(X I-\mu \hat{q}-\nu \hat{p})^{2}}{2 \sigma^{2}}} .
$$

One interesting property, that is preserved by the smoothing of the tomogram, is that the marginals $\mathcal{W}_{\Xi}(X, \mu, \nu)$ are also probability distributions. In the limit $\sigma \rightarrow 0, \Xi(z) \rightarrow \delta(z), \widetilde{\Xi}(-1)=1, \mathcal{N}_{\Xi}=1$.
In the case of multimode systems with quadratic Hamiltonians, the dequantizer and quantizer of thick tomography with a Gaussian weight function $\Xi$ become

$$
\hat{U}_{\sigma}(X, \vec{\mu}, \vec{\nu})=\Xi\left(X \mathbb{I}-\frac{1}{2}(\hat{\vec{Q}}-\vec{r}) \cdot B(\hat{\vec{Q}}-\vec{r})\right)
$$

and

$$
\hat{D}_{\sigma}(X, \vec{\mu}, \vec{\nu})=\frac{|\operatorname{det} B|}{(2 \pi)^{N+1} \tilde{\Xi}(-1)} \mathrm{e}^{\mathrm{i}\left(X \mathbb{I}-\frac{1}{2}(\hat{\vec{Q}}-\vec{r}) \cdot B(\hat{\vec{Q}}-\vec{r})\right)},
$$

respectively. Thus, the quantum thick tomogram of a multimode state with weight function $\Xi$ reads

$$
\begin{aligned}
\mathcal{W}_{\Xi}(X, \vec{\mu}, \vec{\nu})= & \int \frac{d \vec{p} d \vec{q}}{(2 \pi)^{N}} W(\vec{q}, \vec{p}) \\
& \Xi\left(X-\frac{1}{2}(\vec{Q}-\vec{r}) B(\vec{Q}-\vec{r})\right),
\end{aligned}
$$

in terms of the Wigner functions of the state, and the inverse formula

$$
\begin{aligned}
W(\vec{q}, \vec{p})= & \frac{|\operatorname{det} B|}{(2 \pi)^{N+1} \widetilde{\Xi}(-1)} \int d X d \vec{\mu} d \vec{\nu} \mathcal{W}_{\Xi}(X, \vec{\mu}, \vec{\nu}) \\
& \mathrm{e}^{i\left(X-\frac{1}{2}(\vec{Q}-\vec{r}) B(\vec{Q}-\vec{r})\right)}
\end{aligned}
$$

permits to reconstruct the Wigner function from its tomograms.

The proof of the inverse formula follows the same steps of the case of linear symplectic tomograms. Indeed, if we plug (71) into the right hand side of (72) we get

$$
\begin{aligned}
& \frac{|\operatorname{det} B|}{(2 \pi)^{N+1} \widetilde{\Xi}(-1)} \int d X d \vec{\mu} d \vec{\nu} \int \frac{d \vec{p}^{\prime} d \vec{q}^{\prime}}{(2 \pi)^{N}} W\left(\vec{q}^{\prime}, \vec{p}^{\prime}\right) \\
\Xi & \left(X-\frac{1}{2}\left(\vec{Q}^{\prime}-\vec{r}\right) B\left(\vec{Q}^{\prime}-\vec{r}\right)\right) \mathrm{e}^{i\left(X-\frac{1}{2}(\vec{Q}-\vec{r}) B(\vec{Q}-\vec{r})\right)} \\
= & \frac{|\operatorname{det} B|}{(2 \pi)^{N+1} \widetilde{\Xi}(-1)} \int d X d Y d \vec{\mu} d \vec{\nu} \int \frac{d \vec{p}^{\prime} d \vec{q}^{\prime}}{(2 \pi)^{N}} W\left(\vec{q}^{\prime}, \vec{p}^{\prime}\right) \\
& \delta\left(Y-\frac{1}{2}\left(\vec{Q}^{\prime}-\vec{r}\right) B\left(\vec{Q}^{\prime}-\vec{r}\right)\right) \Xi((X-Y) \mathbb{I}) \\
= & \frac{|\operatorname{det} B|}{2 \pi \widetilde{\Xi}(-1)} \int d Z d \vec{p}^{\prime} d \vec{q}^{\prime} W\left(\vec{q}^{\prime}, \vec{p}^{\prime}\right) \int \frac{d \vec{\mu} d \vec{\nu}}{(2 \pi)^{N}} \Xi(Z) \mathrm{e}^{i Z} \\
= & \frac{1}{\widetilde{\Xi}(-1)} W(\vec{q}, \vec{p}) \int \frac{d Z}{2 \pi} \Xi(Z) \mathrm{e}^{i Z}=W(\vec{q}, \vec{p})
\end{aligned}
$$

Let us consider the example of a quadratic thick tomogram governed by a Hamiltonian with

$$
B=\left(\begin{array}{c}
\mathbb{I}_{2}, 0 \\
0, \mathbb{I}_{2}
\end{array}\right)
$$

and Gaussian weight function $\Xi_{\sigma}$ for a system with one degree of freedom. In that case, one might think that the tomogram is centered on a quadratic curve

$$
X=\frac{1}{2}(p-\nu)^{2}+\frac{1}{2}(q-\mu)^{2} .
$$


The tomogram is given by

$$
\begin{aligned}
\mathcal{W}_{\sigma}(X, \mu, \nu)= & \frac{1}{2 \pi \sqrt{2 \pi \sigma^{2}}} \int \mathrm{d} p \mathrm{~d} q W(q, p) \\
& \mathrm{e}^{-\frac{\left(x-\frac{1}{2}(p-\nu)^{2}-\frac{1}{2}(q-\mu)^{2}\right)^{2}}{2 \sigma^{2}}},
\end{aligned}
$$

in terms of the Wigner function and the inverse transform

$$
\begin{aligned}
W(q, p)= & \frac{\mathrm{e}^{\frac{\sigma^{2}}{2}}}{2 \pi} \int \mathrm{d} X \mathrm{~d} \mu \mathrm{d} \nu \mathcal{W}_{\sigma}(X, \mu, \nu) \\
& \mathrm{e}^{\mathrm{i}\left(X-\frac{1}{2}(p-\nu)^{2}-\frac{1}{2}(q-\mu)^{2}\right)},
\end{aligned}
$$

allows a reconstruction of the Wigner function from its thick quadratic tomograms.

\section{COMPARISON WITH THE THICK RADON TRANSFORM}

A comparison with the quantum Radon transform (1) is useful and clarifies the merits of the approach taken in this article. Let us consider the thick version of (11), with a window function $\Xi$

$$
\mathcal{R}_{\Xi}(X, \varphi)=\operatorname{Tr} \hat{\rho} \Xi(X \hat{\mathbb{I}}-\hat{q} \cos \varphi-\hat{p} \sin \varphi) .
$$

Now, in general, relation (5) (a consequence of the positive homogeneity of degree -1 of the Dirac distribution) does not hold anymore for $\mathcal{R}_{\Xi}$ and $\mathcal{W}_{\Xi}$. As a consequence, one has to deconvolve $\mathcal{R}_{\Xi}$-whenever this is possible - in order to get $\mathcal{R}_{\hat{\rho}}$ and thus $\hat{\rho}$. Since

$$
\mathcal{R}_{\Xi}(X, \varphi)=\int \Xi(X-Y) \mathcal{R}_{\hat{\rho}}(Y, \varphi) \mathrm{d} Y,
$$

one gets

$$
\int \mathrm{d} X \mathcal{R}_{\Xi}(X, \varphi) \mathrm{e}^{-\mathrm{i} k X}=\tilde{\Xi}(k) \int \mathrm{d} X \mathcal{R}_{\hat{\rho}}(X, \varphi) \mathrm{e}^{-\mathrm{i} k X} .
$$

Equation (80) can be inverted for any $\mathcal{R}_{\hat{\rho}}$ if and only if $\tilde{\Xi}(k) \neq 0$ for all $k$. In this case, from (80) and (9) one gets

$$
\begin{aligned}
\hat{\rho}= & \frac{1}{2 \pi} \int \mathrm{d} X \int_{0}^{\infty} \mathrm{d} r \int_{0}^{2 \pi} \mathrm{d} \varphi \frac{\mathcal{R}_{\Xi}(X, \varphi)}{\tilde{\Xi}(-r)} \\
& \times r \mathrm{e}^{\mathrm{i} r(X \hat{I}-\hat{q} \cos \varphi-\hat{p} \sin \varphi)} .
\end{aligned}
$$

Therefore, in contrast with the thick symplectic transform, the thick Radon transform cannot be inverted for arbitrary window functions. Moreover, even when the inversion is possible, one needs to have a complete knowledge of the window function in order to deconvolve the thick homodyne tomogram and perform its inversion (81).

\section{CONCLUSIONS}

There are many possible interesting applications of the quantum tomograms introduced in this article by using quadrics and window functions in the phase-space. The measurements of variables that are quadratic forms of positions and momenta are equivalent to measuring the energy of vibrations with changing parameters, like minima of potential energy, elastic forces, and so on. This procedure can be used to measure the state $\hat{\rho}$ of trapped ions by varying the parameters of the trapping potential.

Homodyne detection, by using non-linear crystals as beam splitters, also creates quadratic forms of the photon quadrature components which, in principle, can be measured by varying the parameters of the quadratic forms. Thus, tomography with the generalized quantized version of the Radon transform provides new possibilities to measure quantum states.

From a formal viewpoint, the new tomographic maps and their inverse can be formulated within the framework of the star-product scheme, with quantizers and dequantizers for multidimensional systems. Some of the constructions involve generic quadratic forms of the different position and momentum operators. The inhomogeneous symplectic group of the corresponding multidimensional phase spaces and its subgroups play a significant role in the formalism. The new quantum tomograms can be considered as the quantum version of the generalized classical tomograms introduced in our previous work [32] and involve quadratic surfaces on phase spaces. The correspondence between both approaches is established by means of the Weyl map.

In quantum optics, the new non-linear Radon transforms can be easily extended to the quantum domain by using the Weyl-Wigner map. The results of this article show that the reconstruction of the Wigner function using optical or symplectic tomography based on straight-line Radon transform can be extended to situations in which the marginals in phase space are measured for curved hyperbolas or ellipses. In particular, parabolic tomography could be implemented with the recently observed accelerated Airy beams [38].

Finally, the other generalization introduced in this paper, named "thick" quantum tomography, entails that the effect of a non-singular window function not only smooths the tomograms, but in fact matches better all practical applications, where thick tomograms arise in a natural way. The striking fact we pointed out in this work is that the reconstruction procedure in this case is almost independent on the window functions, with an enormous advantage due to the fact for most experimental devices the window function is not completely known.

\section{Acknowledgments}

V.I.M. was partially supported by the Russian Foundation for Basic Research under Project No. 11-02-00456. 
This work partially supported by a cooperation grant INFN-MICINN. M.A. was also partially supported by the Spanish MICINN grant FPA2009-09638 and DGIIDDGA (grants 2009-2010-2011-E24/2).
[1] W. Pauli, in Handbuch der Physik, Eds. Geiger, Scheel, Vol. XXIV, Part 1, 1933. [Reprinted in Encyclopedia of Physics, Vol. V, T. I (Springer, Berlin 1958)]

[2] W. Pauli, General Principles of Quantum Mechanics, translated by P. Achuthan and K. Venkatesan, page 17 (Allied Publishers Private Limited, Bombay, 1980).

[3] H. Reichenbach, Philosophic Foundations of Quantum Mechanics (University of California Press, Berkeley, 1944).

[4] V. I. Manko, G. Marmo, A. Simoni, A. Stern, F. Ventriglia, Phys. Lett. A 343, 251 (2005).

[5] J. Radon, Über die bestimmung von funktionen durch ihre integralwerte längs dewisse mannigfaltigkeiten, Breichte Sachsische Akademie der Wissenschaften, Leipzig, Mathematische-Physikalische Klasse, 69 S. 262 (1917).

[6] E. P. Wigner, Phys. Rev. 40, 749 (1932).

[7] J. Bertrand and P. Bertrand, Found. Phys. 17, 397 (1987).

[8] K. Vogel and H. Risken, Phys. Rev. A 40, 2847 (1989).

[9] D. T. Smithey, M. Beck, M. G. Raymer, and A. Faridani, Phys. Rev. Lett. 70, 1244 (1993).

[10] J. Mlynek, Phys. Rev. Lett. 77, 2933 (1996).

[11] C. Kurtsiefer, T. Pfau, and J. Mlynek, Nature 386, 150 (1997).

[12] Quantum State Estimation, Lecture Notes in Physics Vol. 649, edited by M. G. A. Paris and J. Řeháček (Springer, Berlin, 2004).

[13] A. I. Lvovsky and M. G. Raymer, Rev. Mod. Phys. 81, 299 (2009).

[14] Z. Hradil, Phys. Rev. A 55, R1561 (1997).

[15] G. Zambra, A. Andreoni, M. Bondani, M. Gramegna, M. Genovese, G. Brida, A. Rossi, and M. G. A. Paris, Phys. Rev. Lett. 95, 063602 (2005).

[16] G. Badurek, et al, Phys. Rev. A 73, 032110 (2006).

[17] P. Banáš, J. Řeháček, and Z. Hradil, Phys. Rev. A 74, 014101 (2006);

[18] Z. Hradil, D. Mogilevtsev, and J. Řeháček, Phys. Rev. Lett. 96, 230401 (2006).

[19] T. Kiesel, W. Vogel, V. Parigi, A. Zavatta and M. Bellini, Phys. Rev. A 78, 021804R (2008).

[20] V. D'Auria, S. Fornaro, A. Porzio, S. Solimeno, S. Olivares and M. G. A. Paris, Phys. Rev. Lett 102, 020502 (2009).

[21] A. Zavatta, V. Parigi, M. S. Kim, H. Jeong and M.
Bellini, Phys. Rev. Lett. 103, 140406 (2009).

[22] A. Allevi, A. Andreoni, M. Bondani, G. Brida, M. Genovese, M. Gramegna, S. Olivares, M. G. A. Paris, P. Traina and G. Zambra, Phys. Rev. A 80, 022114 (2009).

[23] M. A. Nielsen and I. L. Chuang, Quantum Computation and Quantum Information (Cambridge University Press, Cambridge, 2000).

[24] V. Vedral, Introduction to Quantum Information Science (Oxford University Press, Oxford, 2006).

[25] S. G. Mihlin, Multi-dimensional singular integrals and integral equations, Pergamon Press, New York (1965).

[26] I. M. Gel'fand and G. E. Shilov, Generalized Functions: Properties and Operations, Vol. 5 (Academic Press, 1966).

[27] S. Helgason, Ann. of Math. 98, 451 (1973); Groups and Geometric Analysis (Academic Press, Orlando, 1984); The Radon Transform (Birkhauser, Boston, 1980).

[28] S. Mancini, V. I. Man'ko and P. Tombesi, Quantum Semiclass. Opt. 7, 615 (1995).

[29] O. V. Man'ko and V. I. Man'ko, J. Russ. Laser Res. 18, 407 (1997).

[30] M. Asorey, P. Facchi, V.I. Man'ko, G. Marmo, S. Pascazio and E. C. G. Sudarshan, Phys. Rev. A 76, 012117 (2007).

[31] O. V. Man'ko, V. I. Man'ko, G. Marmo, J. Phys. A: Math. Gen. 35, 699 (2002).

[32] M. Asorey, P. Facchi, V. I. Man'ko, G. Marmo, S. Pascazio and E. C. G. Sudarshan, Phys. Rev. A 77, 042115 (2008).

[33] A. S. Arkhipov and V. I. Man'ko, Phys. Rev. A 71, 012101 (2005).

[34] V. I. Man'ko, G. Marmo, A. Simoni, A. Stern and E. C. G. Sudarshan, Phys. Lett. A 35 (2005) 351.

[35] P. Facchi, M. Ligabò and S. Pascazio, J. Mod. Optics, 57, 239 (2010).

[36] M. Asorey, P. Facchi, G. Florio, V. I. Man'ko, G. Marmo, S. Pascazio, E. C. G. Sudarshan, Phys. Lett. A 375, 861 (2011).

[37] A. Ibort, V. I. Manko, G. Marmo, A. Simoni and F. Ventriglia, Phys. Lett. A 374 (2010) 2614.

[38] G. A. Siviloglou, J. Broky, A. Dogariu and D. N. Christodoulides, Phys. Rev. Lett. 99, 213901 (2007). 